\title{
Diffusion model of defect formation in silicon under light ion implantation
}

\author{
M.V. Voznyy, P.M. Gorley, V.A. Schenderovskyy* \\ Yu. Fedkovych Chernivtsi State University, 2, Kotsyubynskyy St., 58012, Chernivtsi, Ukraine \\ Tel.: (380-372)598473,FAX:(380-372)551809,E-mail: horley@ite.cv.ua \\ "Institute of Physics of NASU, 144, Prospect Nauki, 03650, Kyiv, Ukraine \\ Tel.: (380-44)650 777, FAX: (380-44)650 777,E-mail: schender@iop.kiev.ua
}

\begin{abstract}
In the given paper the model of defect formation in silicon under light ion implantation is proposed which describes the whole complex of available experimental results qualitatively, and in a number of cases rather well quantitatively. In contrast to the models existing by now, it takes into account the dissociation processes of complex defects. In the frame of assumption about subsurface vacancy absorption layer existence the expressions for spatial distributions of secondary defect stationary concentrations are obtained using the Lie group theory. Non-stationary complex defect system behavior in silicon is simulated depending on vacancy trap concentration, ion current density and implanted atom energy.
\end{abstract}

Keywords: ion implantation, silicon, secondary defects, diffusion, complexes' dissociation.

Paper received 24.02.00; revised manuscript received 12.05.00; accepted for publication 16.06.00.

\section{Introduction}

During light ion implantation into the crystal the main kinds of generated defects are mobile vacancies $(V)$ and interstitials $(I)$, which can interact with one another (recombining or forming complexes) and also with other defects of the material crystal lattice: impurity atoms, dislocations, surface. The maximum density of introduced primary defects corresponds to the maximum of ion energy losses [1]. The distribution of secondary defects (complexes) determines physical characteristics of irradiated crystal and can be significantly different from the ion energy elastic loss distribution, as far as it depends on many factors, namely: mobility of defects, their interaction parameters, surface influence and so on [2]. In paper [3] under low energy ion implantation into silicon the distributions of vacancy complexes with two-layer defect structure are obtained. Except the maximum corresponding to ion energy elastic losses deep inside the crystal significant subsurface defect concentration is observed in the samples. In [4] on the basis of analysis of experimental results [3], an assumption about subsurface vacancy absorption layer existence was made, and a model of defect formation process in silicon under implantation was proposed. That model, however, doesn't take into account the complicated complex dissociation possibility as it is indicated by experimental results. In the given paper more real diffusion model of defect formation in silicon under light ion implantation is proposed. In addition to taking into consideration complex dissociation reactions, in a number of cases it gives an opportunity to obtain stationary-spatial secondary defect distributions under arbitrary known spatial distributions of primary defects and vacancy traps. Results of theoretical simulations carried out on the basis of the proposed model explain rather successfully the whole variety of experimental data [3].

\section{Model of implantation and stationary solu- tions}

Let us consider that one-dimensional differential model of low-energy ion implantation of silicon is described by the following system of evolution equations:

$$
\left\{\begin{aligned}
v_{t} & =K(x)+m v_{x x}-g i v-a v^{2}+s i w- \\
& -c[N(x)-u] v-p u v-k v w-b v, \\
i_{t} & =K(x)+n i_{x x}-g i v-s i w-r i y-h i, \\
w_{t} & =0.5 a v^{2}-s i w+p u v-k v w+r i y, \\
u_{t} & =c[N(x)-u] v-p u v, \\
y_{t} & =k v w-r i y,
\end{aligned}\right.
$$

where $v, i, w, u, y$-concentrations of vacancies, interstitials, divacancies, trapped vacancies and threevacancies respectively; $m, n$ - primary defect diffusivities; $a, b, c, g$, 


\section{M.V. Voznyy et al.: Diffusion model of defect formation in silicon under light ...}

$h, k, p, r, s$-coefficients of appropriate quasi-chemical reactions; $K(x)$ and $N(x)$ - spatial distributions of generated primary defects and vacancy traps, respectively, $K(x)=j P f(x), j$-ion current density, $P$ - maximum number of defects generated by ion with the given energy, $f(x)$ - ion energy elastic loss normalized distribution, $x$-spatial coordinate ( $x I[0, L]$, where $L$-sample length), $t$-time, subindexes stand for appropriate derivatives.

The essential difference between model (1) and those proposed in [4] and [5] is that in (1) the possibility of complex dissociation reactions is taken into consideration $(s \neq 0, r \neq 0)$.

Let us use mathematical apparatus of the Lie group theory [6] for determination the solution of system (1). With the aid of group analysis [6], it can be easily shown that infinitesimal operators generating admittable Lie groups for (1) are the following:

$$
\left\{\begin{aligned}
X_{1}=\partial_{t}, \quad \forall b, h, K(x), N(x), \\
X_{2}=\partial_{x}, \quad \forall b, h ; K(x)=K_{0}, N(x)=N_{0}, \\
X_{3}=\left(A_{1} x+A_{2}\right) \partial_{x}+2 A_{1}\left(t \partial_{t}-v \partial_{v}-\right. \\
\left.\quad-i \partial_{i}-w \partial_{w}-u \partial_{u}-y \partial_{y}\right), \\
b=h=0, \quad K(x)=K_{0}\left(A_{1} x+A_{2}\right)^{-4}, \\
N(x)=N_{0}\left(A_{1} x+A_{2}\right)^{-1},
\end{aligned}\right.
$$

where $K_{0}, N_{0}, A_{1}, A_{2}$ are constants.

Furthermore, the conservation law for the total number of monovacancies can be indicated (interstitials are considered here to be monovacancies with the negative sign). Differential form of this law has a form:

$(v-i+2 w+u+3 y)_{t}=m v_{x x}-n i_{x x}-b v+h i$

Considering the complex formation as a main factor in defect system stability achievement [4], we can evaluate the influence of unsaturable traps as not essential $(b=$ $h=0$ ) and obtain an integral form of the conservation law from (3):

$$
\int_{0}^{L}(v-i+2 w+u+3 y)_{t} d x=\left.m v_{x}\right|_{0} ^{L}-\left.n i_{x}\right|_{0} ^{L} .
$$

It is stated by (4) that change of the total number of monovacancies in the crystal is determined exclusively by the fluxes of primary defects through the sample boundaries.

As it follows from formulae (2), the only operator generating the solutions for the function $K(x)$ with maximum located inside the crystal is $X_{1}=\partial_{t}$. The corresponding invariant set $\{x, v, i, w, u, y\}$ leads to stationary solutions of system (1). In the absence of primary defect flow through the sample, the differential form of total monovacancy number conservation law can be obtained from (3) and (4) (due to arbitrary value of $L$ ). $n i_{x x}=m v_{x x}$.

Direct integration of system (1) taking into account expression (5) leads to the following stationary distributions of defects in the crystal:

$$
\left\{\begin{array}{l}
i_{0}(x)=m v_{0}(x) / n+C_{1} x+C_{2}, \\
u_{0}(x)=c N(x) /(c+p), \\
w_{0}(x)=\left[2 s i_{0}(x)\right]^{-1}\left[a v_{0}^{2}(x)+2 p v_{0}(x) u_{0}(x)\right], \\
y_{0}(x)=\left[r i_{0}(x)\right]^{-1}\left[k v_{0}(x) w_{0}(x)\right],
\end{array}\right.
$$

where $C_{1}, C_{2}$ are constants, defined from the boundary conditions, and $v_{0}(x)$ is a solution of the second-order ordinary differential equation:

$$
\begin{aligned}
& m\left(v_{0}\right)_{x x}=\left(\frac{a v_{0}^{2}}{2}+\frac{c p N(x) v L_{0}}{c+p}\right) \times \\
& \times\left(1+\frac{k v_{0}}{s i_{0}(x)}\right)+g v_{0} i_{0}(x)-K(x),
\end{aligned}
$$

which can be integrated numerically with arbitrary $K(x)$ and $N(x)$.

\section{Results and disscusion}

Integration of the equation system (6) and (7) was performed using the fourth-order Runge-Kutta procedure [7] for given forms of primary defect distribution functions [2], [4]:

$$
K(x)=K_{0} \exp \left[-\left(\frac{x-x_{0}}{l_{1}}\right)^{2}\right]
$$$$
N(x)=N_{0} \exp \left(-x / l_{2}\right) \text {. }
$$

Parameter values $K_{0}, N_{0}, x_{0}, l_{1}$ and $l_{2}$ in (8) were determined from experimental data [2], [4]. Results of theo-

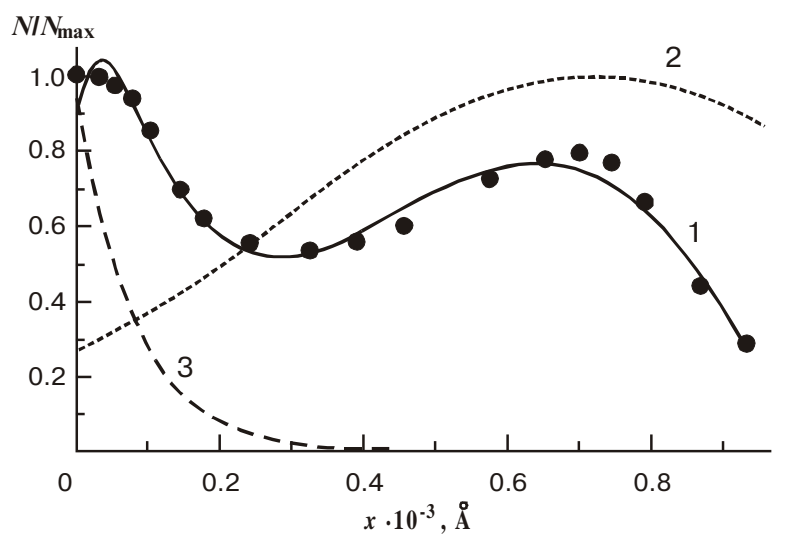

Fig. 1. Spatial defect distributions in Si implanted by $\mathrm{B}^{+}$-ions: 1 threevacancy concentration $y(x)$ according to the model (1) (points are experimental results for profiles of $V V$-centers [3]); 2 - primary defect generation rate $K(x) ; 3$ - absorption layer density $N(x) ; j=1 \mu \mathrm{A} / \mathrm{cm}^{2}, E=40 \mathrm{keV} \mathrm{[3].}$

$S Q O, 3(3), 2000$ 

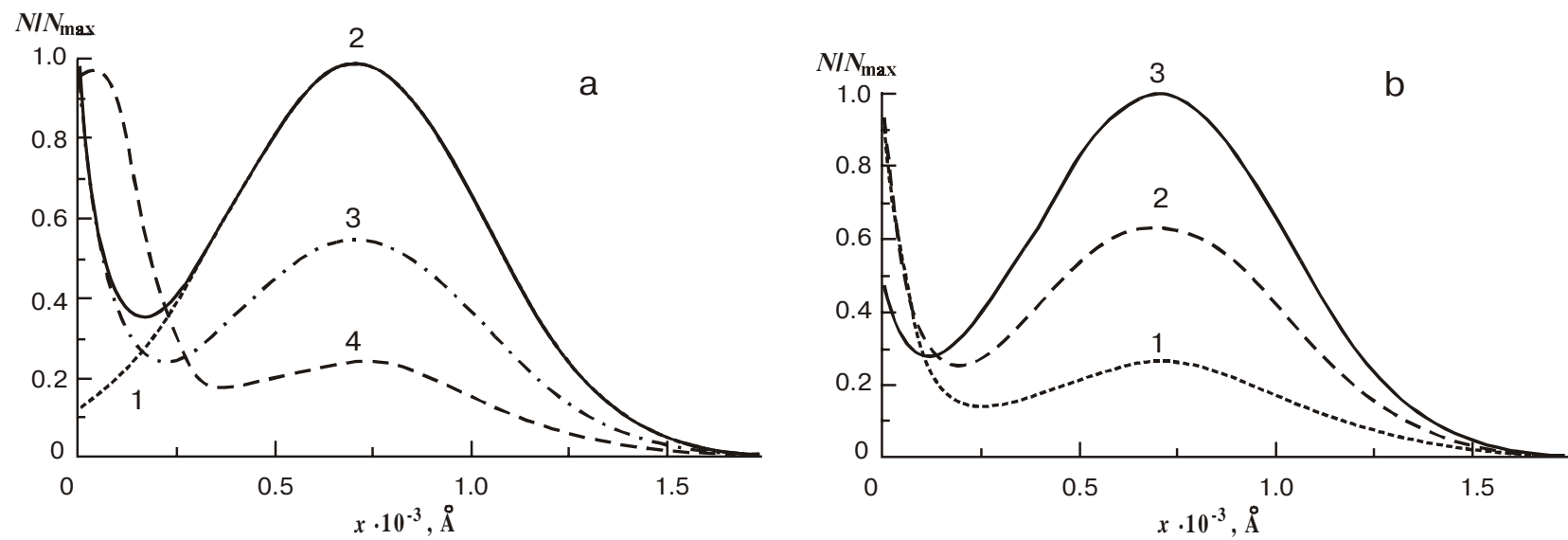

Fig. 2. Calculated threevacancy profiles under implantation as dependent on:

a) vacancy trap concentration $N(x)$, ion current density $j$ is constant $\left(K_{0}=0,6\right)$ : $1-N_{0}=0 ; 2-N_{0}=0,03 ; 3-N_{0}=0,15 ; 4-N_{0}=0,75$.

b) ion current density $j$, trap concentration is constant $\left(N_{0}=0,15\right)$ :

$1-K_{0}=0,06 ; 2-K_{0}=0,6 ; 3-K_{0}=6$.

retical simulations for $y(x)$-distribution and experimental data [3] are presented in Fig. 1. It is easy to see that proposed model (1) of defect formation in silicon in stationary case describes the experimental data rather successfully. The relative error between theoretical and experimental results does not exceed $5 \%$. It allows on the basis of assumptions of system (1) to carry out a behavior prediction of dynamic system of defects in silicon as dependent on the values of operating parameters (ion current density, ion energy and trap concentrations). During such simulations the solutions of system (1) were determined using net method [8].

In Fig. 2 normalized to peak concentrations distributions are presented as dependent on vacancy trap concentration $N(x)$ and ion current density $j$. In the absence of traps the single maximum is present (curve 1, Fig. 2a).

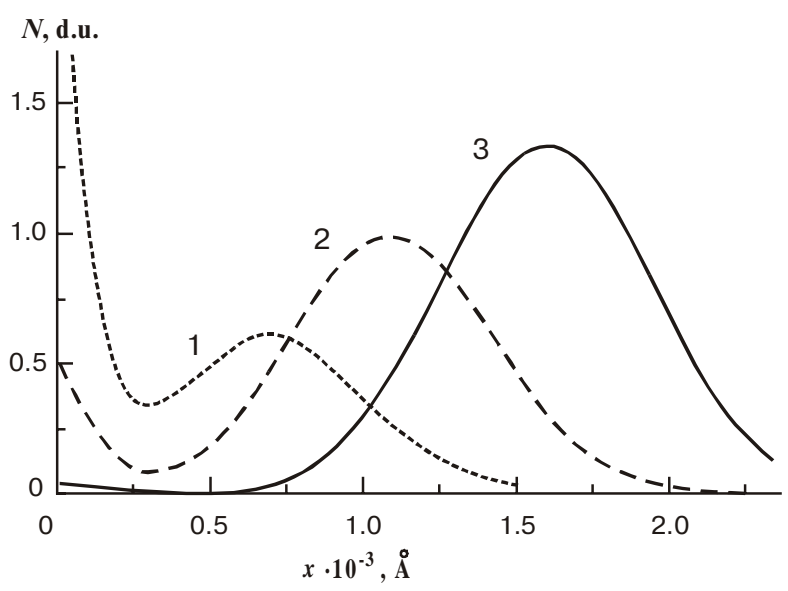

Fig. 3. Spatial threevacancy distributions in dependence on bombarding ion energy $E$ :

$1-40 \mathrm{keV} ; 2$ - $52 \mathrm{keV} ; 3-76 \mathrm{keV}$.
When trap concentration increases (curves 2-4, Fig. 2a) formation of subsurface defected layer is observed (the second peak appears on the plot). Moreover, secondary defect concentration in subsurface layer can be much greater than that in the ion energy elastic loss area. Inverse system behavior is obtained when ion current density increases under constant trap concentration (Fig. 2b). Deep peak becomes more noticeable and ultimately can exceed significantly the subsurface level.

Secondary defect distribution dependence on energy of bombarding atoms is shown in Fig. 3. It should be noted that even significant subsurface defect concentration can decrease almost to zero when ion energy increases only twice. It can be explained by the location of energy elastic loss maximum further from sample surface (to 0.1 micron deeper), while in subsurface layer (0.02-0.05 micron) [4] secondary defects are generated less effectively due to lack of free vacancies in this area. Thus, the subsurface vacancy absorption layer existence can lead according to experiment [3] to formation of defected layer of secondary defects under low current density and low energy of bombarding ions or to its absence under another conditions.

Dose dependence of threevacancy concentration is shown in Fig. 4. It can be seen that taking into consideration the complex dissociation reactions $(s>0, r>0)$ leads to almost constant threevacancy concentration after definite (high enough) dose (curve 1, Fig. 4). But if dissociation processes are absent then saturation for the concentration of the most complicated defects in the system is not achievable [4], [5], and it will increase linearly with dose ultimately (curves 2 and 3, Fig. 4). In that case from (6) and (7) only the form of spatial distribution for threevacancy concentration can be determined but not the exact values of this concentration. The exact form of secondary defect distribution profiles under arbitrary irra- 


\section{M.V. Voznyy et al.: Diffusion model of defect formation in silicon under light ...}

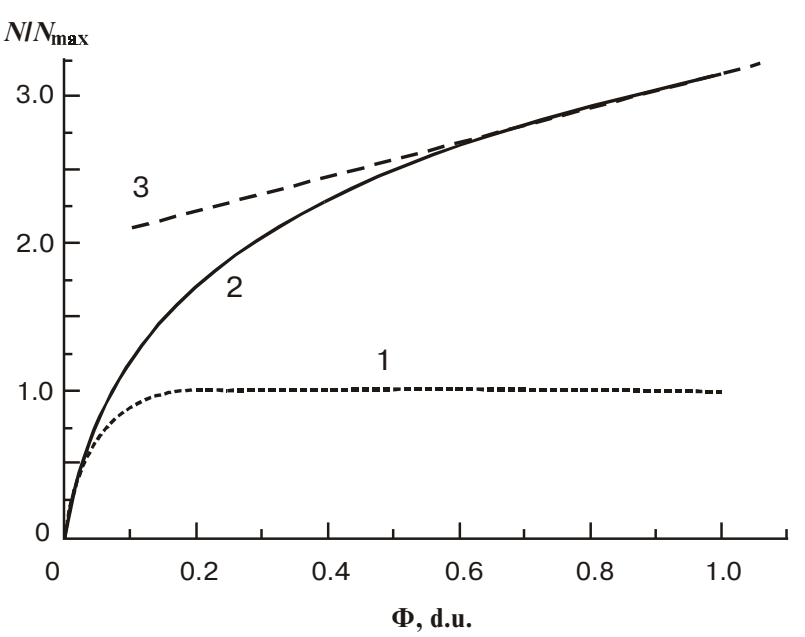

Fig. 4. Threevacancy concentration dependence on irradiation dose $\Phi$ according to the model (1):

1 - defect dissociation is considered; 2 - processes of dissociation are not taken into account, 3 - linear concentration increase under complex defect accumulation [5].

Note: abbreviation «d.u.» on figures stands for «dimensionless units».

diation dose can be obtained in that case using net methods of numerical integration of the equation system (1).

\section{Conclusions}

Thus, the model of radiation defect formation under implantation of silicon with light ions is proposed in the paper, which takes into consideration complex dissociation processes and enabled us for the first time to explain the whole available variety of experimental results qualitatively (and in some cases quantitatively). For the given model, using the Lie group theory the stationary solution is obtained, which can be used for determination of speed constants of quasi-chemical reactions as well as for determination of the form of primary defects and vacancy trap distributions on the basis of available experimental data. Theoretical results obtained allow predicting of the system behavior of secondary defects in silicon as dependent on specific values of ion current density, parameters of subsurface absorption layer and implanted ion energy.

\section{References}

1. I. A. Abroyan, A. N. Andronov, A. I. Titov, Physical principles of electronic and ionic technology, Vysshaya shkola, Moscow (1984) (in Russian).

2. V. L. Vinetskiy, G. A. Kholodar, Radiation physics of semiconductors, Naukova dumka, (1979) (in Russian).

3. V. N. Gashtold, N. N. Gerasimenko, A. V. Dvurechenskiy , L.S. Smirnov, Profiles of defects under implantation of ions into silicon // Fizika i Tehnika Poluprovodnikov 9(5), pp.835839 (1975) (in Russian).

4. A. V. Kondrachuk, A. I. Petropavlovskiy, V. Z. Iordanishvili, Model of defect formation in semiconductor under light ion implantation // Ukrainskiy Fizicheskiy Zhurnal 35(10), pp.1573-1579 (1990) (in Russian).

5. N. P. Morozov, D. I. Tetelbaum, P. V. Pavlov, E. I. Zorin, Diffusion-coagulation model of radiation defect accumulation under ion bombarding of silicon // Fizika $i$ Tehnika Poluprovodnikov 9(12), pp. $2292-2295$ (1975) (in Russian).

6. P. J. Olver, Applications of Lie groups to differential equations, Graduate texts in mathematics, Springer-Verlag, Berlin, Heidelberg, New York, Tokyo (1986).

7. Ya. T. Grinchishin, V. I. Efimov, A. N. Lomakovich, Algorithms and programs on BASIC, Prosveschenie, Moscow (1988) (in Russian).

8. W. E. Milne, Numerical solution of differential equations, New York, London (1953). 